\title{
A computational approach for optimizing vehicles' interior noise and vibration
}

\section{Adel Mohammed Al-Dhahebi ${ }^{1}$, Ahmad Kadri Junoh ${ }^{1}$, Zamri Mohamed ${ }^{2}$ and Wan Zuki Azman Wan Muhamad ${ }^{1}$}

\author{
${ }^{1}$ Institute of Engineering Mathematics, Universiti Malaysia Perlis, \\ 02600, Kampus Pauh Putra, Arau Perlis, Malaysia \\ ${ }^{2}$ Faculty of Mechanical Engineering, Universiti Malaysia Pahang, \\ 26600 Pekan Pahang, Malaysia \\ E-mail: aldhahebi.adel@gmail.com \\ Phone: +60-111-9122212
}

\begin{abstract}
This paper proposes a Genetic Algorithm (GA) to optimise vehicles' interior noise and vibration caused by powertrain, tire-road surface interaction and type of car. Toward this end, an experimental design was carried out to obtain the noise and vibration data of three local compact-sized cars at stationary and running conditions and varying engine speeds. The acquired data were analysed to obtain sound quality parameters such as loudness and sharpness, sound pressure level and vibration exposures in the interior cabin. Besides that, a K-means clustering algorithm was utilised to cluster the noise and vibration to determine the comfort level in the vehicle's interior cabin. The overall findings indicate that the comfort level is influenced by the types of road surface, powertrain and vehicle design. The results also indicate that the proposed GA approach is reliable and can be utilised by automotive researchers to identify the optimal Noise, Vibration and Harshness (NVH) values for vehicle refinement and noise control.
\end{abstract}

Keywords: K-means clustering algorithm; Genetic Algorithm; Interior Vehicle Noise and Vibration Optimization; Sound Quality; Structure Vibrations; Experimental Designs of NVH.

\section{INTRODUCTION}

Vehicle noise and vibration is a key attribute to car quality and reliability and it can influence customers' decisions in buying a car [1-4]. Since the 1980s, automotive noise and vibration has received tremendous attention by researchers and has become an essential factor in vehicle design and validation process. The main sources of vehicle noise, vibration and harshness $(\mathrm{NVH})$ that were identified consist of the tyre-road noise which dominates in the medium and high-speed ranges, the powertrain noise [5] which is dominant in low to medium speed ranges, and the wind noise that is dominant in high speed ranges [1]. The interior NVH can be classified into air-borne noise and structureborne noise based on their transmission to the passenger cabin. The structure-borne noise is caused by a vibrating source (i.e. the engine structural vibrations) that induces acoustic energy to travel through solid structures and then released as air-borne noise [6]. In the case of noise due to tyre-road interaction, vibration energy is structurally transmitted through the chassis and suspension where it then radiates from the body panels into the passenger compartment. The repetitive exposure to structural vibration (hand, foot and 
backrest) for long periods can lead to Whole Body Vibration (WBV) [7, 8]. For the last two decades, minimizing the interior's NVH has been a key focus of research in the automotive industry. Various methods have been applied, including psychoacoustic, subjective assessments and active or passive noise cancelation techniques [9-16]. Other studies have employed shock absorbers and computational fluid mechanics in hybrid cars to improve ride comfort $[17,18]$. However, recent demands and competitiveness have forced the automotive industry to strive for more economical and lightweight designs which has resulted in making the vehicle's interior invariably noisier due to the increased level of structural vibration. In an attempt to resolve the aforementioned problem, various testing and assessment methods have been developed to characterise and assess the noise and vibration in the interior cabin. It is a great challenge for sound quality engineers to match customers' expectations towards vehicle cabin noise which brings about physical and subjective evaluations. Another concern on sound quality is that different car types have different levels of comfort as perceived by customers. From luxury cars to compact cars, different sound qualities will be expected and thus, require further investigation in terms of sound classification based on the car segment. In this case, bias in perception will occur since every user will have a pre-set view of comfort rating based on their perspective and daily exposure. The characteristics of a vehicle's interior sound can be described as informative as it implies a certain image and may identify similar optical impressions [19]. To this end, multiple researchers have employed psychoacoustic metrics in an attempt to evaluate the vehicle's interior noise and vibration and thus its sound quality such as in [3, 9-12, 15, 20-27].

Recent researches in Malaysia have focused on investigating the NVH in Proton sedan cars while neglecting compact cars. For example, Nore et al. [10] developed a comfort index called the Vehicle Acoustical Comfort Index (VACI) to evaluate the sound quality and comfort level in the interior cabin of the Proton Perdana V6 by combining subjective and objective measurements. VACI has been applied extensively in various studies including $[13,28]$. In another study, Nopiah et al. [29] proposed a method to cluster and classify the level of sound quality (noise and vibrations) into categories according to their comfort level in vehicles' interior cabin. Meanwhile, Nopiah et al. [28] proposed a goal-programming approach to optimise the comfort level in Proton sedan cars by considering the interior noise caused by the powertrain NVH. Recently, Nopiah et al. [13] proposed a hybrid fuzzy nonlinear weighted goal programming that optimises the acoustics level in the Proton Perdana sedan V6 cabin. In another study, Nopiah et al. [30] assessed the vehicle's interior sound quality (noise and vibration) using hybrid classification and clustering techniques including neural networks, hierarchical clustering and Linear Discriminant Analysis (LDA). Other studies have investigated the vertical Whole-Body Vibration (WBV), and steering and engine performances such as in $[8,31$, 32]. Based on the above discussion and literature, it must be pointed out that sound quality differs in different automotive vehicles and thus, the existing models do not provide reliable solutions to the noise and vibration phenomena. Instead, the proposed models are valid for certain types of vehicles and under specific testing conditions and considerations. Thus, it is extremely difficult to completely eliminate vehicle interior noise; however, it can be controlled and optimised. Hence, based on previous studies, the methods employed to evaluate interior noise should be investigated further. In light of these, an accurate experimental evaluation and optimization of vehicles' (compact-sized) $\mathrm{NVH}$ at both idle and running conditions is essential in order to undertake an accurate refinement of vehicle sound quality. Such procedures should measure noise and vibration concurrently to determine the effects of structural vibrations on vehicles' overall sound 
quality. Besides, the literature indicates limited studies on the noise and vibration of compact-sized cars' interiors, particularly in Malaysia.

Hence, this paper intends to propose an experimental design to acquire sound and vibration signals using three local compact cars namely Myvi, Viva and Axia which have not been explored on in previous studies. The purpose is to identify, characterise and validate the noise and vibration sources in order to provide suggestions for improvement in the vehicles' interior sound comfort. Moreover, this paper proposes an optimisation method using the Genetic Algorithm to optimise the noise and vibration in vehicles' interior cabin. GA is an effective heuristic approach which enables the searching for the optimal noise and vibration values to minimise vehicle interior noise. The purpose of developing the GA model is to determine the extent to which vibration exposures influence the noise (loudness, sone) level in the interior of a vehicle's cabin. The GA model considers loudness as the control variable, while vibration values act as the independent variable.

\section{METHODS AND MATERIALS}

\section{Experimental Design}

The experimental design was developed to identify variables which are known to influence noise and vibration in vehicles' interior cabin. We defined three main influences which are powertrain, road surface and type of cars. The tested compact-sized cars have the following features; auto transmission, year 2015, engine of 1.0 and tyre sizes of 175/65R14, 175/65R14 and 165/55R14 for Axia, Myvi and Viva respectively. The sound and vibration signals were measured using National Instruments hardware and software (NI cDAQ-9174, NI-9234, NI-DAQmx, and LabVIEW). Sounds and vibrations were recorded using the GRAS microphone and IMI accelerometer respectively.

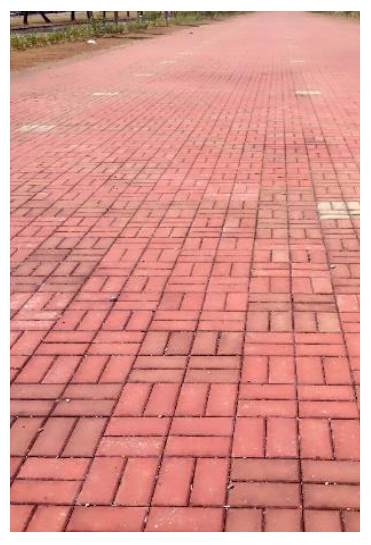

a) Pavement Road

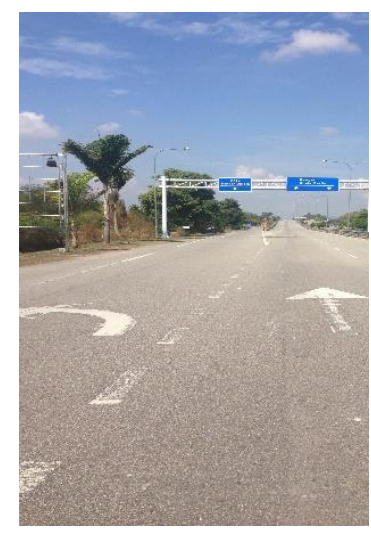

b) Highway Road

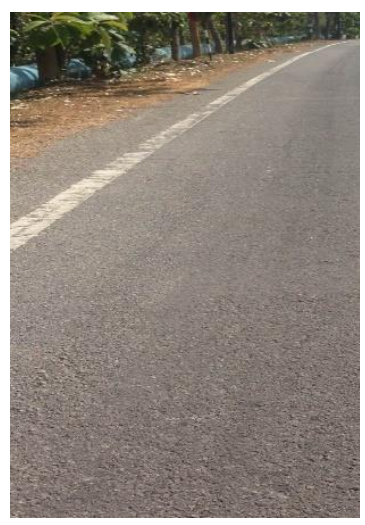

c) Urban Road

Figure 1.Tested road surfaces. location: Arau, Perlis, Malaysia.

Measurements were carried out under four driving conditions namely pavement, highway, urban and idle as illustrated in Figure 1. The duration of measurement was set to 10 seconds and the experiments were repeated seven (7) times in order to ensure the statistical reliability and accuracy of data because encountered errors may occur during the tests. The sound and vibration signals were measured against changes of engine speed 
rpm at 1000, 1250, 1500, 1750, 2000 and 2250. Four running criteria were imposed, including the appropriate placement of microphones and accelerometers, sealed windows, usage of air conditioning, and manual control for the wheel and engine speeds according to the test plan. Furthermore, the tests were done at night to minimize ambient noise. Aerodynamic noise was ignored as its contribution is small at low vehicle speeds. The microphone and accelerometers locations are illustrated in Figures 2 and 3 respectively.



Figure 2. Microphone Placement: Front Dashboard

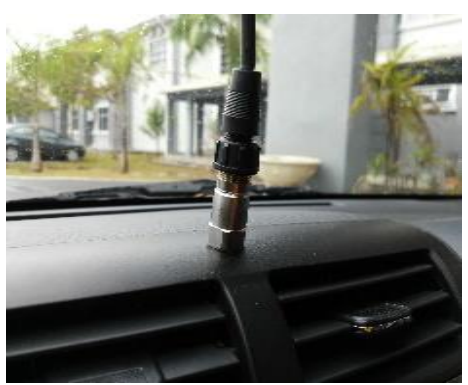

a) Front Dashboard



b) Car Floor

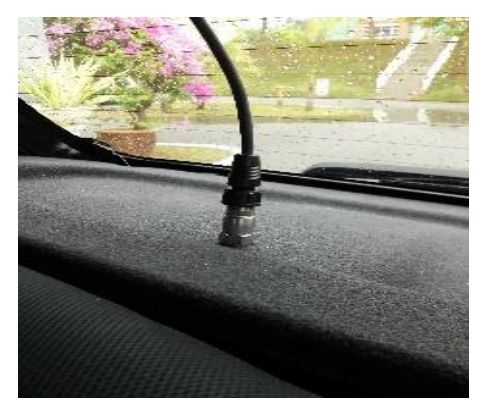

c) Back Dashboard

Figure 3. Accelerometer placements.

\section{Noise and Vibration Evaluation}

The most efficient noise assessment method is the psychoacoustics Zwicker objective metrics loudness (sone) and sharpness (acum) [33, 34]. They bring about the sound assessment without the need to perform any tedious subjective assessment tests. These objective measures are intended to describe the sounds experienced in the interior cabin efficiently. For example, loudness aims at characterising humans' awareness towards the strength of the perceived sounds. In this study, the Sound Quality Software Bruel \& Kjaer (B \& K) type 7698 was utilised to analyse the recorded sounds in order to obtain the sound quality's Zwicker loudness and sharpness with respect to different engine speeds, road surfaces and car types. In addition, sound quality parameters are observed against sound pressure levels in all 7 recordings. Some examples of sound quality loudness and sharpness for Axia on a pavement road surface and at an engine speed of $2000 \mathrm{rpm}$ are described in Figure 4. 


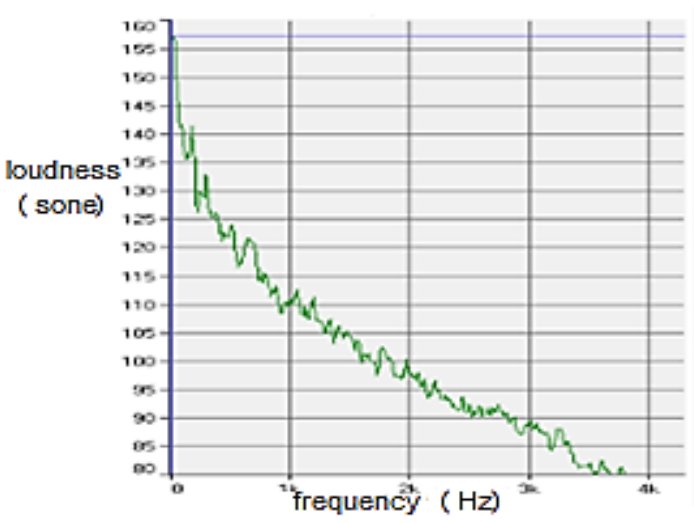

a) Loudness (Sone)

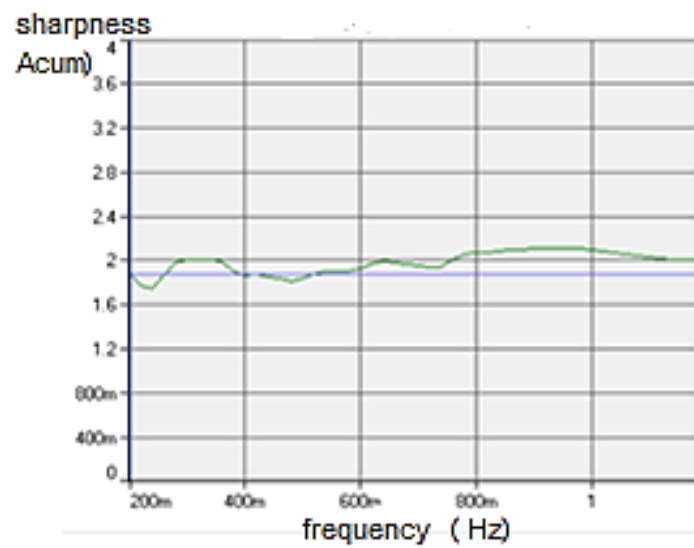

b) Sharpness (Acum)

Figure 4. The sound quality metric loudness and sharpness (acum) of Axia on a pavement road surface at $2000 \mathrm{rpm}$.

For vibration evaluation, ISO 2631 and the BS 6841 were used as reference where the vibration severity estimation was taken from the axis that has the highest vibration. The Root Mean Square (RMS) method was used to assess the total vibration exposure to passengers and drivers within the given time duration. The method is mathematically expressed in Equation (1) (ISO 2631).

$$
a(R M S)=\left({ }_{-}^{1} \int_{0}^{T} a(t)^{2} d t\right)^{1 / 2}
$$

where

$a(R M S)$ is a value of the acceleration $\left(\mathrm{m} / \mathrm{s}^{2}\right)$

$a(t)$ represents the measured acceleration

$T$ represents the time period (in seconds) of exposed vibration.

\section{Interior Comfort Clustering}

Clustering is a multivariate iterative process used to simplify the large noise and vibration database in order to obtain useful and meaningful information to describe the comfort level in a vehicle cabin. The K-means algorithm is developed in this study using MATLAB software to cluster the comfort level. The reason behind selecting it is that it provides accurate and tight clustering. Here, the comfort level is clustered into 5 categories as described in Table 1. In this case, the clustering of vibrations was conducted with respect to the trends of sound quality loudness in the vehicle interior cabin. The purpose of clustering is to determine the impact of both sounds and vibrations concurrently on the overall comfort in the interior of the vehicle cabin. The process of grouping the noise and vibration data is through their distances from each other, and is called Euclidean distances. This distance refers to the distance between the noise and vibration points and the corresponding centroid. During the process of clustering, after the number of clusters is specified, the k-means selects a set of $\mathrm{n}$ points known as clusters seeds as the first guess of the mean of the clusters. Each observation is assigned to the nearest seed to form a set of temporary clusters. After that, the seeds are replaced by cluster means, and the points are reassigned. The process continues until no further 
changes occur in the clusters. The flowchart of the k-means clustering algorithm is displayed in Figure 5.



Figure 5. K-means clustering algorithm flowchart.

Table 1. Clustering categories.

\begin{tabular}{cc}
\hline Level of Comfort & Class \\
\hline Very comfortable & 1 \\
Fairly comfortable & 2 \\
Adequate disturbance & 3 \\
Slight and intrusive disturbance & 4 \\
Extremely and emotionally & 5 \\
disturbing & \\
\hline
\end{tabular}

\section{Genetic Algorithm}

The Genetic Algorithms (GA) model was developed in this study to optimise the noise and vibration in the interior cabins of three compact-sized cars, namely Axia, Myvi and Viva. The GA model aims to search for promising noise and vibration values that produce the minimum loudness value and thus, achieve the optimal comfort level. The GA model considers loudness as the control variable, while the vibration values act as the independent variable. To optimise noise and vibration, six chromosomes were identified in which each chromosome represents one point of vibration. In this case, the search for an optimal value goes through the six chromosomes that represent the vibration exposures. At the start, the initial vibration population was generated by following the rules of max and min values of vibrations as the purpose is to determine optimal vibration values through the assessed vibration exposure points. Next is the reproduction step which is to select chromosomes from the population to be parents for the new chromosomes, and entering them into the mating pool. The parent chromosomes' selection is done according to the min and max vibration values. Next, each new generation of chromosomes were formed through reproduction, crossover and mutation on the old 
population. Finally, the offspring chromosomes were compared with the population pool ones via their fitness values to derive a new population where the weak chromosomes are eliminated or replaced with a child chromosome from the offspring pool. The fitness of the GA is based on the loudness identified in the early evaluation of the measured sound. To sum up, the complete optimisation procedures of the GA approach are described in the following points:

i. Step 1: Generate an initial population of $N$ solutions and identifying the size and length of the selected chromosomes. The probabilities of the crossover $p_{c}$ and the mutation $p_{m}$ are also identified in this step.

ii. Step 2: Evaluate each solution of the initial population using a fitness function/objective function. This evaluation serves to measure the performance of the process and creates the base for the chromosome selection process which will be mated during the reproduction stage.

iii. Step 3: Select solutions as parents for the new generation based on probability or randomness. The best solutions (in terms of fitness or objective) have a higher probability to be selected than poor solutions.

iv. Step 4: Use the parent solutions from Step 3 to produce the next generation (called offspring). This process is called as crossover. The offsprings are placed in the initial set of solutions to replace the weaker solutions.

v. Step 5: Randomly alter the new generation through mutation. Usually, this is done using a mutation probability.

vi. Step 6: Repeat Step 2 through Step 5 until the stopping criterion is met.

vii. Step 7: Replace the parent (initial) chromosome populations with the offspring (new) populations.

viii. Step 8: Go to Step 4, and repeat the process until the criteria of termination is satisfied. The termination criteria can be realised by either the number of iterations or when a specific objective function is achieved. In this study, the GA coding is made to run 10 times for 5000 iterations, and the results are then averaged to determine the minimum sound quality value and thus, optimal comfort. The crossover rate was set to $25 \%$ and the mutation rate to $75 \%$. The GA coding model was conducted for every tested engine speed according to the road surface type and car.

\section{RESULTS AND DISCUSSION}

The results of sound quality loudness and sharpness as well as psychoacoustic sound pressure levels for Viva, Myvi and Axia on a highway road surface are presented in Table 2. The displayed results are the average of seven recorded runs at different engine speeds rpm. It can be observed in Table 2 that there are significant increases in loudness, sharpness and sound pressure values with increases in engine speed. Besides, the type of road surface is a major contributor to the overall sound quality in the interior cabin. Pavement road surfaces produce higher noise (loudness and sharpness) than highway and urban road surfaces. This can be due to the irregularities and uneven structures of pavement road surfaces which cause a louder noise in the vehicle cabin [30]. Meanwhile, due to the smoothness and even structure of the highway road surface, less amount of noise is produced in the three tested cars [30]. As for the urban road surface, the amount of noise is less than that of the pavement road surface. For idle conditions, the interior noise is high due to the increase of engine speed while the car is stationary, as shown by 
the results in Table 2. Apart from this, it can be observed that the type of car influences the amount of noise produced on the highway, pavement, urban and idle driving conditions. Although there is not much difference between the noises in the three tested compact cars, Axia has the highest comfort compared to Myvi and Viva in both stationary and non-stationary conditions.

Table 2. Sound Quality Results of Cars Tested on the Highway Road Surface

\begin{tabular}{lccc}
\hline rpm & Sound Pressure $(\mathrm{dB})$ & Loudness (Sone) & Sharpness (Acum) \\
\hline \multicolumn{4}{c}{ Viva } \\
\hline 1000 & 1848 & 3788 & 1.518 \\
1250 & 2300 & 5150 & 1.617 \\
1500 & 3734 & 4490 & 1.567 \\
1750 & 3497 & 4084 & 1.530 \\
2000 & 3540 & 5155 & 1.614 \\
2250 & 3880 & 4974 & 1.606 \\
\hline \multicolumn{5}{c}{ Myvi } \\
\hline 1000 & 1360 & 2045 & 1.656 \\
1250 & 2002 & 2254 & 1.207 \\
1500 & 1642 & 3537 & 1.474 \\
1750 & 1862 & 3385 & 1.451 \\
2000 & 2258 & 3818 & 1.503 \\
2250 & 2455 & 4141 & 1.544 \\
\hline \multicolumn{5}{c}{ Axia } \\
\hline 1000 & 746 & 2045 & 1.656 \\
1250 & 814 & 2254 & 1.207 \\
1500 & 1534 & 3537 & 1.474 \\
1750 & 1475 & 3385 & 1.451 \\
2000 & 1811 & 3818 & 1.503 \\
2250 & 1771 & 4141 & 1.544 \\
\hline
\end{tabular}

Meanwhile, the average results for the vibration evaluation on the highway road surface for Axia, Myvi and Viva are presented in Table 3. Based on these results, it can be observed that the vibration exposures are influenced by the changes in engine speed (rpm) as well as the type of road surface and car. When the engine speed (rpm) is increased, the vibration exposure in the interior vehicle cabin also increases as shown by the results in Table 3. In addition, the type of road surface impacts the vibration in the vehicle interior cabin in which the pavement road surface produces the highest vibration level compared to highway and urban road surface. However, the values of vibration exposure at idling conditions are higher than at running conditions as the increase in engine speed while the car is in a stationary condition contributes to higher noise as indicated by the results in Table 2. As such, Axia has the lowest vibration dose among the three cars. Meanwhile, the results of the comfort clustering for Axia, Myvi and Viva on the highway road surface are presented in Table 4. It can be observed that Axia has the most comfortable interior cabin when under highway, pavement, urban and idle driving conditions. Interior comfort in this case is described based on the clustered loudness and vibration exposures in the interior cabin of the three tested cars. 
Table 3. Vibration Exposures of Cars on the Highway Road Surface $\left(\mathrm{m} / \mathrm{s}^{2}\right)$.

\begin{tabular}{cccccccc}
\hline Car & rpm & $\begin{array}{c}\text { Front dash } \\
\text { box }\end{array}$ & $\begin{array}{c}\text { Front floor } \\
\text { left }\end{array}$ & $\begin{array}{c}\text { Front floor } \\
\text { right }\end{array}$ & $\begin{array}{c}\text { Rear-door } \\
\text { left }\end{array}$ & $\begin{array}{c}\text { Rear-door } \\
\text { right }\end{array}$ & $\begin{array}{c}\text { Back dash } \\
\text { box }\end{array}$ \\
\hline \multirow{4}{*}{ Axia } & 1000 & 0.003974 & 0.001776 & 0.001888 & 0.002419 & 0.002021 & 0.003186 \\
& 1250 & 0.005925 & 0.002675 & 0.002277 & 0.003188 & 0.002769 & 0.004141 \\
& 1500 & 0.005814 & 0.003049 & 0.003134 & 0.004728 & 0.004107 & 0.005918 \\
& 1750 & 0.006291 & 0.004218 & 0.003779 & 0.005154 & 0.004431 & 0.007294 \\
& 2000 & 0.006808 & 0.005107 & 0.003853 & 0.005434 & 0.004964 & 0.007171 \\
& 2250 & 0.007435 & 0.004336 & 0.004420 & 0.005639 & 0.005368 & 0.008866 \\
\cline { 2 - 8 } Myv & 1000 & 0.005208 & 0.003761 & 0.006703 & 0.003020 & 0.003693 & 0.008073 \\
& 1250 & 0.004204 & 0.003212 & 0.006551 & 0.003190 & 0.003458 & 0.007526 \\
& 1500 & 0.007890 & 0.004560 & 0.010680 & 0.003400 & 0.005301 & 0.007720 \\
& 1750 & 0.009270 & 0.005908 & 0.010338 & 0.004340 & 0.005177 & 0.002056 \\
& 2000 & 0.008213 & 0.009273 & 0.014474 & 0.007130 & 0.010285 & 0.009379 \\
& 2250 & 0.006212 & 0.007863 & 0.011298 & 0.006920 & 0.007531 & 0.007829 \\
\cline { 2 - 8 } Viva & 1000 & 0.006304 & 0.004206 & 0.003225 & 0.003532 & 0.003173 & 0.008684 \\
& 1250 & 0.008602 & 0.005412 & 0.003814 & 0.004149 & 0.003931 & 0.006681 \\
& 1500 & 0.005841 & 0.006865 & 0.005021 & 0.006398 & 0.004992 & 0.004636 \\
& 1750 & 0.050920 & 0.006477 & 0.004706 & 0.006220 & 0.005115 & 0.009656 \\
& 2000 & 0.007200 & 0.008679 & 0.005552 & 0.006737 & 0.006706 & 0.005575 \\
& 2250 & 0.009220 & 0.007276 & 0.004983 & 0.005344 & 0.005384 & 0.004965 \\
\hline \multirow{6}{*}{ i }
\end{tabular}

Table 4. Average clustering results for cars on the highway road surface.

\begin{tabular}{cccccccccc}
\hline Cluster & Cluster & Count & $\begin{array}{c}\text { Loudness } \\
\text { (Sone) }\end{array}$ & $\begin{array}{c}\text { Front } \\
\text { dash } \\
\text { box }\end{array}$ & $\begin{array}{c}\text { Front } \\
\text { floor } \\
\text { left }\end{array}$ & $\begin{array}{c}\text { Front } \\
\text { floor } \\
\text { right }\end{array}$ & $\begin{array}{c}\text { Rear } \\
\text { floor } \\
\text { left }\end{array}$ & $\begin{array}{c}\text { Rear } \\
\text { floor } \\
\text { right }\end{array}$ & $\begin{array}{c}\text { Back } \\
\text { dash } \\
\text { board }\end{array}$ \\
\hline \multirow{3}{*}{ Axia } & 1 & 13 & 2083 & 0.00405 & 0.00194 & 0.00181 & 0.00247 & 0.00223 & 0.00327 \\
& 2 & 6 & 3218 & 0.00593 & 0.00295 & 0.00291 & 0.00403 & 0.00363 & 0.00539 \\
& 4 & 10 & 4082 & 0.00751 & 0.00421 & 0.00423 & 0.00573 & 0.00519 & 0.00843 \\
& 5 & 7 & 3214 & 0.00679 & 0.00416 & 0.00384 & 0.00487 & 0.00436 & 0.00699 \\
& 1 & 4 & 4095 & 0.00831 & 0.00521 & 0.00508 & 0.00663 & 0.00621 & 0.01015 \\
\hline \multirow{3}{*}{ Myvi } & 2 & 12 & 4238 & 0.00592 & 0.00295 & 0.00290 & 0.00403 & 0.00363 & 0.00539 \\
& 3 & 10 & 5162 & 0.00750 & 0.00420 & 0.00423 & 0.00573 & 0.00518 & 0.00843 \\
& 4 & 9 & 3912 & 0.00679 & 0.00415 & 0.00384 & 0.00487 & 0.00436 & 0.00699 \\
& 5 & 6 & 5596 & 0.00831 & 0.00521 & 0.00508 & 0.00663 & 0.00621 & 0.01015 \\
\cline { 2 - 9 }$y$ & 1 & 1 & 6190 & 0.00404 & 0.00194 & 0.00181 & 0.00247 & 0.00225 & 0.00327 \\
Viva & 2 & 13 & 5377 & 0.00592 & 0.00295 & 0.00290 & 0.00403 & 0.00363 & 0.00539 \\
& 3 & 8 & 6807 & 0.00750 & 0.00421 & 0.00422 & 0.00573 & 0.00518 & 0.00843 \\
& 4 & 11 & 10100 & 0.00679 & 0.00415 & 0.00383 & 0.00487 & 0.00436 & 0.00699 \\
& 5 & 9 & 3802 & 0.00831 & 0.00522 & 0.00508 & 0.00663 & 0.00621 & 0.01015 \\
\hline
\end{tabular}

The highway road surface produced the highest comfort values as indicated by "Comfortable" and "Very comfortable" clusters for Axia, Myvi and Viva respectively. Meanwhile, the pavement road surface produced the lowest comfort which can also be described by the number of clusters in the "Noisy" and "Very noisy" clusters [29]. It can be concluded that the type of road certainly influences the interior comfort level in Axia, Myvi and Viva. As for the idle driving condition, the level of comfort depends on the increase of engine speed while the car is in a stationary condition (with zero excitation of 
tires with the road surface). In fact, comfort in this case depends on the design of the vehicle structure, mainly the engine and mechanical components. In this case, the results indicate that the interior comforts of Axia, Myvi and Viva in idle conditions ares in a comfortable range.

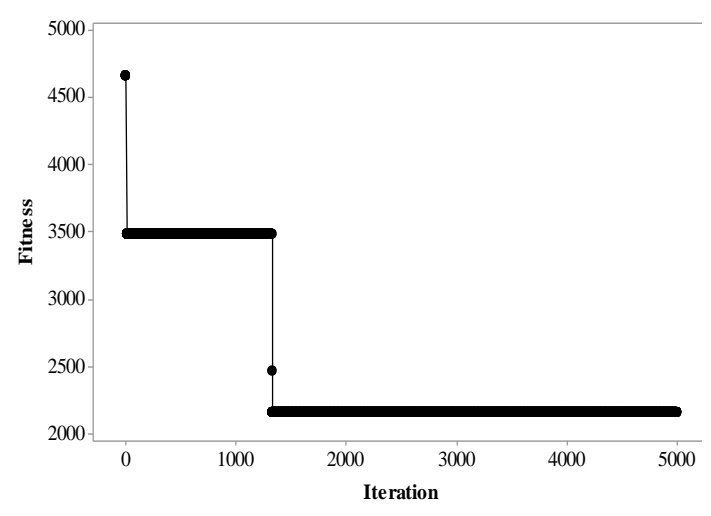

(a) Axia

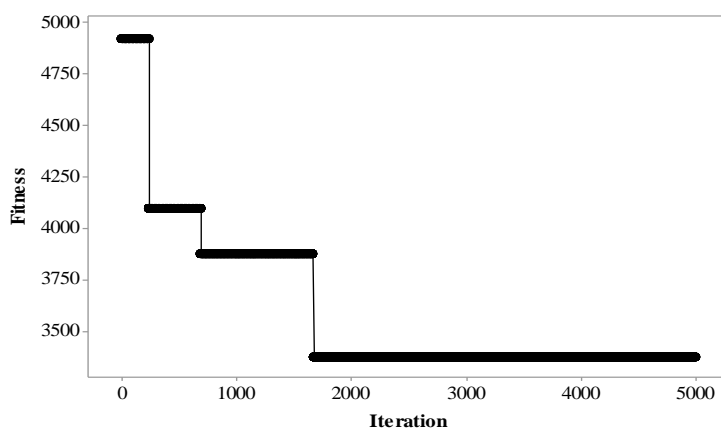

(b) Myvi

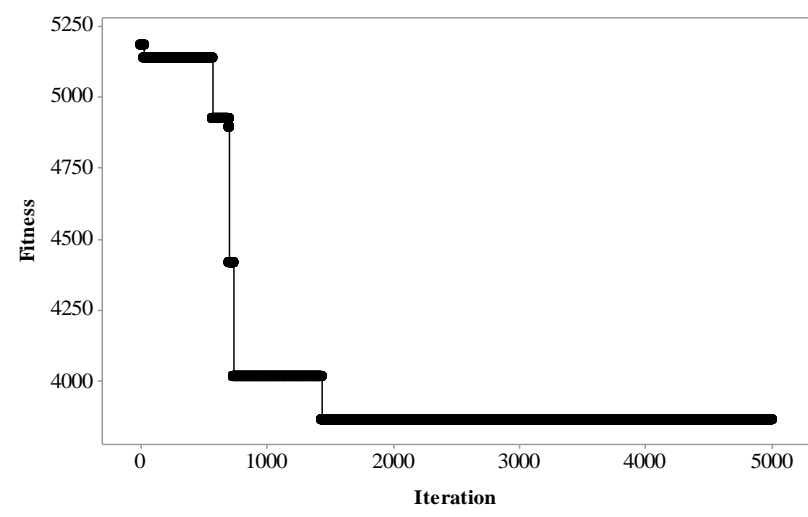

(c)Viva

Figure 6. Graphs representing the fitness functions of the genetic algorithm for Axia, Myvi and Viva on the highway road surface.

Apart from this, Figure 6 shows the scatterplot of the iteration and fitness of the GA model of Axia, Myvi and Viva on the highway road surface. Throughout these graphs, the search for the optimal values of loudness (fitness) using the GA model was limited to 5000 iterations (stopping criteria of GA model). At this point, the values for loudness and vibration exposures were chosen as the optimal solution by the GA model. Those figures represent the $10^{\text {th }}$ run of the GA model. Moreover, the average results of the genetic algorithm optimization model for Axia, Myvi and Viva according the highway, pavement, urban and idle conditions are summarized in Table 5. Based on the GA results, the optimal values of vibration exposures which produce minimum noise can be described by the fitness (loudness) of the GA model. When comparing the obtained loudness values with the ones measured and analysed in the section "Evaluation of Noise and Vibration", we can conclude that the GA model is reliable and accurate. The GA results indicate that Axia has the most comfortable interior cabin when under highway, pavement, urban and idle driving conditions as determined by the amount of loudness (fitness) produced as shown in Table 5 and Figure 7.

Table 5. Optimization model results for cars tested under different driving conditions. 


\begin{tabular}{lllllllll}
\hline Condition & Car & $\begin{array}{l}\text { Front- } \\
\text { dash box }\end{array}$ & $\begin{array}{l}\text { Front- } \\
\text { floor left }\end{array}$ & $\begin{array}{l}\text { Front- } \\
\text { floor } \\
\text { right }\end{array}$ & $\begin{array}{l}\text { Rear- } \\
\text { door left }\end{array}$ & $\begin{array}{l}\text { Rear- } \\
\text { door } \\
\text { right }\end{array}$ & $\begin{array}{l}\text { Back- } \\
\text { dash box }\end{array}$ & $\begin{array}{l}\text { Fitness } \\
\text { (Sone) }\end{array}$ \\
\hline \multirow{3}{*}{ Highway } & Axia & 0.00021 & 0.00106 & 0.00026 & 0.00076 & 0.00153 & 0.00085 & 2161 \\
& Myvi & 0.00121 & 0.00231 & 0.00308 & 0.00180 & 0.00125 & 0.00164 & 3369 \\
& Viva & 0.00229 & 0.00174 & 0.00055 & 0.00453 & 0.00679 & 0.00446 & 3860 \\
& Axia & 0.00393 & 0.00475 & 0.00312 & 0.00298 & 0.00543 & 0.01338 & 2675 \\
Pavement & Myvi & 0.00219 & 0.00069 & 0.00247 & 0.00093 & 0.00033 & 0.00352 & 3921 \\
& Viva & 0.00351 & 0.00264 & 0.01011 & 0.00773 & 0.00214 & 0.00138 & 4880 \\
\multirow{5}{*}{ Urban } & Axia & 0.00222 & 0.00793 & 0.00035 & 0.00717 & 0.00643 & 0.00058 & 1605 \\
& Myvi & 0.00077 & 0.00288 & 0.00380 & 0.00587 & 0.00829 & 0.00030 & 2970 \\
& Viva & 0.00229 & 0.00184 & 0.00370 & 0.00294 & 0.00722 & 0.00533 & 3853 \\
& Axia & 0.00286 & 0.00129 & 0.00102 & 0.00110 & 0.00059 & 0.00161 & 893 \\
& Myvi & 0.00220 & 0.00187 & 0.00296 & 0.00315 & 0.00207 & 0.00231 & 1740 \\
& Viva & 0.00223 & 0.01045 & 0.00055 & 0.00495 & 0.00820 & 0.00560 & 2130 \\
\hline
\end{tabular}

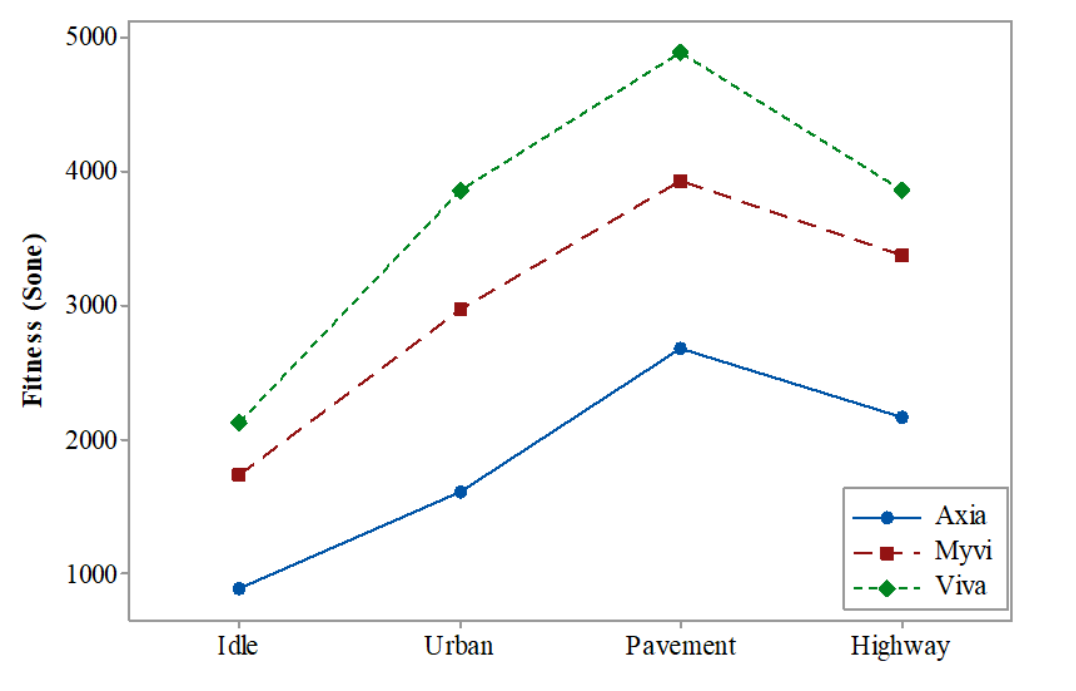

Figure 7. Line Plots for Axia, Myvi and Viva's fitness on the highway, pavement, urban and idle conditions.

In addition, the pavement road surface produces the highest loudness values (fitness), while the highway road surface produces the lowest volume (Figure 7). This means that the type of road and tyres influences the amount of comfort in the interior vehicle cabin as well [28]. At idling conditions, the GA model produces less loudness due to the lower contribution from engine transmission, mechanical accessories, and tyre-road as seen in the results included in Table 5. Thus, it can be observed that Axia on idle has a quieter cabin compared to Myvi and Viva. Apart from this, Figure 6 shows the scatterplot of the iteration and fitness of the GA model of Axia, Myvi and Viva on the highway road surface. Throughout these graphs, the search for the optimal values of loudness (fitness) using the GA model was limited to 5000 iterations (stopping criteria of GA model). At this point, the values for loudness and vibration exposures were chosen as the optimal solution by the GA model. Those figures represent the $10^{\text {th }}$ run of the GA model. Moreover, the average results of the genetic algorithm optimization model for Axia, Myvi and Viva according the highway, pavement, urban and idle conditions are summarized in Table 5. Based on the GA results, the optimal values of vibration exposures which produce minimum noise can be described by the fitness (loudness) of the GA model. 
When comparing the obtained loudness values with the ones measured and analysed in the section "Evaluation of Noise and Vibration", we can conclude that the GA model is reliable and accurate. The GA results indicate that Axia has the most comfortable interior cabin when under highway, pavement, urban and idle driving conditions as determined by the amount of loudness (fitness) produced as shown in Table 5 and Figure 7 . In addition, the pavement road surface produces the highest loudness values (fitness), while the highway road surface produces the lowest volume (Figure 7). This means that the type of road and tyres influences the amount of comfort in the interior vehicle cabin as well [28]. At idling conditions, the GA model produces less loudness due to the lower contribution from engine transmission, mechanical accessories, and tyre-road as seen in the results included in Table 5. Thus, it can be observed that Axia on idle has a quieter cabin compared to Myvi and Viva.

\section{CONCLUSIONS}

This paper's main contribution is the proposed Genetic Algorithm that can be used to optimise compact-sized cars' interior noise and vibration caused by powertrain, tyre-road surface interaction, and type of car. Psychoacoustic objective assessments and sound quality software were employed to obtain the sound pressure level, loudness and sharpness. The interior vibration was assessed using the ISO 2631 Root Mean Square method. Furthermore, this study investigated sound quality and vibration exposures in the interior cabin under different parameters - type of road surface, engine speed and type of car. The results showed that the sound quality and thus the comfort in the vehicle interior cabin are influenced 'by these parameters. Conclusively, Axia has the best sound quality and comfort level compared to Myvi and Viva on the highway, pavement and urban roads at both idling and driving conditions as indicated by the k-means clustering algorithm and the GA model. The proposed Genetic Algorithm model produces optimal noise and vibration values and is a promising method for further research.

\section{ACKNOWLEDGEMENT}

This research was supported in part by Universiti Malaysia Perlis with two short term grants: (9001-00534 and 9001-00533). The authors would like to express their appreciation for the above fund support.

\section{REFERENCES}

[1] Qatu MS. Recent research on vehicle noise and vibration. International Journal of Vehicle Noise and Vibration. 2012;8:289.

[2] Panza MA. A Review of Experimental Techniques for NVH Analysis on a Commercial Vehicle. Energy Procedia. 2015;82:1017-23.

[3] Zhang E, Hou L, Shen C, Shi Y, Zhang Y. Sound quality prediction of vehicle interior noise and mathematical modeling using a back propagation neural network (BPNN) based on particle swarm optimization (PSO). Measurement Science and Technology. 2016;27.

[4] Wang YS, Guo H, Feng TP, Ju J, Wang XL. Acoustic behavior prediction for lowfrequency sound quality based on finite element method and artificial neural network. Applied Acoustics. 2017;122:62-71. 
[5] Zouani A, Hanim S. Overview of noise and vibration in automotive engines. International Journal of Vehicle Noise and Vibration. 2016;12:162-81.

[6] Hanouf ZA, Faris WF. Investigation into noise problems in vehicle structure using vibro-acoustic approach. International Journal of Vehicle Noise and Vibration. 2009;5:238-60.

[7] Wang.X. Vehicle Noise and Vibration Refinement. London, UK: Elsevier, Woodhead Publishing.

[8] Nawayseh N. A mathematical model of the apparent mass of the human body under fore-and-aft whole-body vibration. International Journal of Automotive and Mechanical Engineering. 2016;13:3613-27.

[9] Lee SK. Objective evaluation of interior sound quality in passenger cars during acceleration. Journal of Sound and Vibration. 2008;310:149-68.

[10] Nor MJM, Fouladi MH, Nahvi H, Ariffin AK. Index for vehicle acoustical comfort inside a passenger car. Applied Acoustics. 2008;69:343-53.

[11] Wang X, Subic A. Psychoacoustic modelling of vehicle side mirror power-fold actuator noise characteristics. Proceedings of the Institution of Mechanical Engineers, Part C: Journal of Mechanical Engineering Science. 2011;225:141929.

[12] Mao J, Hao Z, Wang X, Zheng X, Jing G. Loudness simulation and optimization of an internal combustion engine. Neiranji Xuebao/Transactions of CSICE (Chinese Society for Internal Combustion Engines). 2014;32:78-83.

[13] Junoh AK. Hybrid fuzzy k-nearest neighbour technique for assessing the level of interior acoustics in vehicle cabin. International Journal of Vehicle Noise and Vibration. 2015;11:225-37.

[14] Egab L, Wang X. Objective evaluation of interior trim effects on sound quality and noise reduction of a coupled plate cavity system. Mechanical Systems and Signal Processing. 2016;70:919-31.

[15] Kane PV, Andhare AB. Application of psychoacoustics for gear fault diagnosis using artificial neural network. Journal of Low Frequency Noise, Vibration and Active Control. 2016;35:207-20.

[16] Yoon J-H, Yang I-H, Jeong J-E, Park S-G, Oh J-E. Reliability improvement of a sound quality index for a vehicle HVAC system using a regression and neural network model. Applied Acoustics. 2012;73:1099-103.

[17] Barethiye VM, Pohit G, Mitra A. Analysis of a quarter car suspension system based on nonlinear shock absorber damping models. International Journal of Automotive and Mechanical Engineering. 2017;14:4401-18.

[18] Ramasamy D, Mohanesan K, Kadirgama K, Noor MM, Rahman MM. Hybrid electric vehicle car body drag analysis using computational fluid dynamics. International Journal of Automotive and Mechanical Engineering. 2017;14:4496507.

[19] Genuit K. The sound quality of vehicle interior noise: a challenge for the NVHengineers. International Journal of Vehicle Noise and Vibration. 2004;1:158-68.

[20] Akeroyd MA. The psychoacoustics of binaural hearing: La psicoacústica de la audición binaural. International journal of audiology. 2006;45:25-33.

[21] Leite RP, Paul S, Gerges SNY. A sound quality-based investigation of the HVAC system noise of an automobile model. Applied Acoustics. 2009;70:636-45.

[22] Shin S-H, Ih J-G, Hashimoto T, Hatano S. Sound quality evaluation of the booming sensation for passenger cars. Applied Acoustics. 2009;70:309-20. 
[23] Brizon CJdS, Bauzer Medeiros E. Combining subjective and objective assessments to improve acoustic comfort evaluation of motor cars. Applied Acoustics. 2012;73:913-20.

[24] Wang YS, Shen GQ, Guo H, Tang XL, Hamade T. Roughness modelling based on human auditory perception for sound quality evaluation of vehicle interior noise. Journal of Sound and Vibration. 2013;332:3893-904.

[25] Hohls S, Biermeier T, Balschke R, Becker S. Psychoacoustic analysis of HVAC noise with equal loudness. INTER-NOISE and NOISE-CON Congress and Conference Proceedings. Melbourne, Australia: Australian Acoustical Society ( AAS ); 2014. p. 1800-6.

[26] Wang YS, Shen GQ, Xing YF. A sound quality model for objective synthesis evaluation of vehicle interior noise based on artificial neural network. Mechanical Systems and Signal Processing. 2014;45:255-66.

[27] Huang HB, Li RX, Huang XR, Yang ML, Ding WP. Sound quality evaluation of vehicle suspension shock absorber rattling noise based on the Wigner-Ville distribution. Applied Acoustics. 2015;100:18-25.

[28] Nopiah ZM, Junoh AK, Ariffin AK. A novel hybrid fuzzy nonlinear weighted goal programming for optimising interior acoustics level in car cabin. Journal of Vibration and Control. 2013;21:1721-44.

[29] Nopiah ZM, Junoh AK, Ariffin AK. K-means clustering and neural network for evaluating sound level vibration in vehicle cabin. Journal of Vibration and Control. 2013;21:1698-720.

[30] Nopiah ZM, Junoh AK, Ariffin AK. Vehicle interior noise and vibration level assessment through the data clustering and hybrid classification model. Applied Acoustics. 2015;87:9-22.

[31] Aalizadeh B, Asnafi A. Integrated bees algorithm and artificial neural network to propose an efficient controller for active front steering control of vehicles. International Journal of Automotive and Mechanical Engineering. 2016;13: 347691.

[32] Varma M, Kumar R, Prasanthi G. An experimental investigation of vibration characteristics of compression ignition engine running with neem methyl ester. International Journal of Automotive \& Mechanical Engineering. 2016;13:343450.

[33] Fastl H. The psychoacoustics of sound-quality evaluation. Acta Acustica united with Acustica. 1997;83:754-64.

[34] Fastl H, Zwicker E. Psychoacoustics: Facts and models. 3rd ed. Berlin: Springer Verlag; 2006. 